\title{
Dissolution Enhancement of Artemisinin with $\beta$-Cyclodextrin
}

\author{
Mitali KAKRAN, ${ }^{a}$ Nanda Gopal SAHOO, ${ }^{a}$ Lin Li, ${ }^{*, a}$ and Zaher JudEH $*, b$ \\ ${ }^{a}$ School of Mechanical and Aerospace Engineering, Nanyang Technological University; 50 Nanyang Avenue, Singapore \\ 639798, Singapore: and ${ }^{b}$ School of Chemical and Biomedical Engineering, Nanyang Technological University; 62 \\ Nanyang Drive, Singapore 637459, Singapore. \\ Received January 12, 2011; accepted January 31, 2011; published online February 1, 2011
}

The main objective of this research is to improve the dissolution rate of artemisinin (ART) by fabrication with $\beta$-cyclodextrin $(\beta-C D)$ as a hydrophilic carrier. Artemisinin nanoparticles and ART/ $\beta$-CD complexes were successfully fabricated by means of evaporative precipitation of nanosuspension. Characterization of the samples was done by scanning electron microscopy (SEM), Fourier transform infrared (FT-IR), X-ray diffraction (XRD), differential scanning calorimetry (DSC) and dissolution tester. Percent dissolution efficiency, mean dissolution time, relative dissolution and similarity factor were calculated for the statistical analysis of dissolution data. FTIR showed some interaction between ART and $\beta-C D$, which can be due to the formation of some ART/ $\beta$-CD complexes. XRD study indicated the presence of two polymorphs of ART, i.e. orthorhombic and triclinic form. Original ART particles and ART nanoparticles fabricated were orthorhombic whereas the free ART in the ART/ $\beta$-CD complexes (not forming complex with $\beta$-CD) was of triclinic form. The crystallinity of ART reduced and more and more $A R T / \beta-C D$ complexes were formed with increasing concentration of $\beta$-CD as indicated by the DSC, XRD and FT-IR studies. Artemisinin nanoparticles and ART/ $\beta$-CD complexes showed significantly faster dissolution than the pure drug due to smaller size (larger surface area), formation of the inclusion complex with $\beta-C D$, formation of the triclinic form for remaining free ART (not forming complex with $\beta$-CD), and amorphous state formation. Evaporative precipitation of nanosuspension was able to successfully fabricate artemisinin in the nanoparticles and complex forms with significantly faster dissolution rates than that of the original artemisinin. The two polymorphic forms of ART were also fabricated and studied.

Key words artemisinin; orthorhombic; triclinic; $\beta$-cyclodextrin; dissolution

Artemisinin, extracted from the Chinese herb Artemisia annua, has an antimalarial activity and is very efficient against multidrug resistant strains of Plasmodium falciparum malaria and cerebral malaria. ${ }^{1,2}$ In addition, the anticancer activity of artemisinin (ART) has also been reported. ${ }^{3)}$ ART is a sesquiterpene lactone (Fig. 1), and the main characteristic structure of ART is the endoperoxide $(\mathrm{C}-\mathrm{O}-\mathrm{O}-\mathrm{C})$ group contained in the trioxane ring, ${ }^{4)}$ which is essential for the pharmaceutical activity of ART. The intestinal permeability of ART is good but it has a low aqueous solubility, which leads to poor absorption after oral intake. Therefore, a major challenge for the pharmaceutical industry is to maximize the absorption of ATR through enhancement of its dissolution velocity.

The dissolution pace of such a poorly soluble drug can be improved by increasing the exposed surface area of the drug particle available for dissolution, which can be achieved by reducing the particle size to nanoscale, ${ }^{5)}$ use of high energy amorphous state, ${ }^{6,7)}$ use of inclusion compounds such as cyclodextrins, ${ }^{8)}$ etc. Beta-cyclodextrin $(\beta-\mathrm{CD})$ is a cyclic oligosaccharide and contains seven glucopyranose rings as shown in Fig. 2a. The cyclodextrin molecule is of toroidal form as shown in Fig. 2b, and has a small cylinder with a hydrophobic central cavity and a hydrophilic exterior. The poorly water-soluble drug molecules can interact with the lipophilic cavity and form a complex. Cyclodextrins are able to form inclusion complexes with lipophilic drugs and have shown to increase the dissolution of some poorly water-soluble drugs such as paclitaxel, quercetin etc. ${ }^{9)}$ The improvement of ART dissolution using cyclodextrins has been previously studied. ${ }^{810)}$ Cyclodextrin-ART complexes were prepared using a slurry method at a molar ratio of $1: 1$. The complex showed a better dissolution rate with enhanced

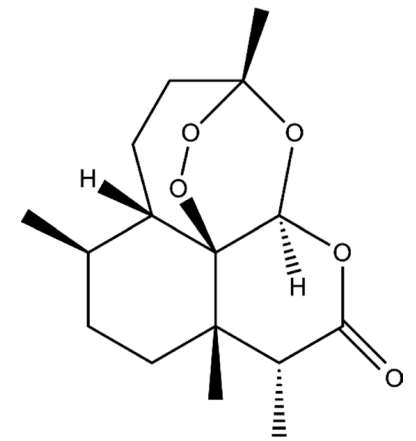

Fig. 1. Chemical Structure of Artemisinin

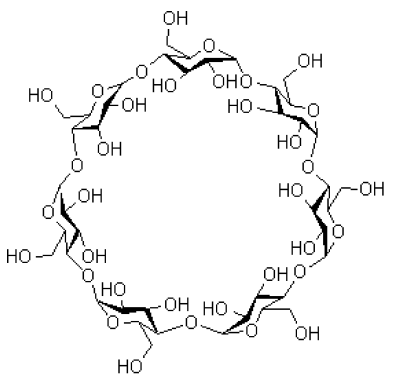

(a)

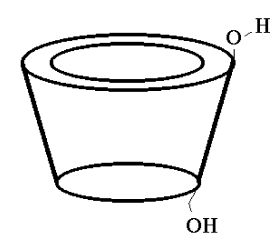

(b)
Fig. 2. (a)Chemical Structure of $\beta$-Cyclodextrin, a Cyclic Oligosaccharide with 7 Glucopyranose Rings; (b) $\beta$-Cyclodextrin as a Small Cylinder with a Hydrophobic Central Cavity

in-vivo bioavailability compared with the commercial drug. Besides that, composite particles of ART with a hydrophilic polymers like polyethylene glycol ${ }^{11)}$ and polyvinylpyrrolidone $^{12)}$ have also been shown to enhance the drug dissolu- 
tion.

The polymorphic form of a drug can have a considerable effect on its pharmaceutical effectiveness, particularly when the dissolution rate is the absorption determining factor in the gastrointestinal tract. Previously Chan et al. reported the X-ray crystallographic evidence for the existence of the two ART polymorphs-triclinic and orthorhombic polymorphs. ${ }^{13}$ ) Both these polymorphs have been fabricated and studied in this work.

In the present study, we aim to prepare artemisinin nanoparticles along with artemisinin/ $\beta$-CD complexes. Evaporative precipitation of nanosuspension (EPN) has been employed to prepare ART nanoparticles and ART/ $\beta$-CD complexes. The interesting findings about the changes in the morphology and crystal structure of ART upon fabrication with $\beta-C D$ as carrier during the EPN fabrication process have been reported. The effect of varying the concentration of $\beta$-CD on ART has also been studied. The dissolution study for all the samples has been conducted and the results have been statistically analyzed to study the influence of different ART to $\beta$-CD ratios on the fabrication and dissolution of artemisinin.

\section{Experimental}

Materials Artemisinin (molecular weight $(\mathrm{MW})=282.332 \mathrm{~g} / \mathrm{mol}$ ) was purchased from Kunming Pharmaceutical Corporation (Kunming, China). $\beta$-Cyclodextrin ( $M W=1134.98)$ was obtained from Sigma Aldrich, Singapore. All reagents used were of technical grade.

Method Artemisinin (ART) nanoparticles and complexes with $\beta$-CD were fabricated by the evaporative precipitation of nanosuspension (EPN) method. The solution of pure artemisinin (ART) was prepared in ethanol and the nanosuspension was obtained by mixing hexane. The drug concentration was $15 \mathrm{mg} / \mathrm{ml}$ in ethanol and the ethanol to hexane ratio was $1: 20$ (by volume). The nanosuspension was evaporated quickly to produce the drug nanoparticles, which were later vacuum dried. This method has demonstrated to yield the smallest particle size and the highest dissolution of ART. ${ }^{14)}$ The same preparation conditions were used to prepare the ART and $\beta$-CD complexes. Different ratios of ART to $\beta$-CD used in the complexes were $1: 1,1: 2$ and $1: 4$ (by weight).

Particle Morphology A scanning electron microscope (JSM-6390LASEM, Jeol Co., Tokyo, Japan) was used to observe the morphology of samples.

Fourier Transform Infrared (FT-IR) Analysis DIGILAB FTS 3100 system was used to perform the FT-IR spectroscopic measurements of the samples.

Differential Scanning Calorimetric (DSC) Analysis The Perkin Elmer DSC 7 thermal analyzer was used to carry out the DSC measurements for the various samples. All the samples weighing $2 \mathrm{mg}$ were used for the DSC tests. The temperature range of $30-250^{\circ} \mathrm{C}$ and heating rate of $10^{\circ} \mathrm{C} / \mathrm{min}$ in nitrogen gas were used. The melting point and heat of fusion were calculated using the DSC software.

X-Ray Diffraction Analysis The X-ray diffractometer (Bruker AXS D8 Advance X-ray diffractometer) was used to study the X-ray diffraction of the samples. $\mathrm{Cu} K \alpha$-targets at a scanning rate of $0.0102 \theta / \mathrm{s}$ and $40 \mathrm{kV}, 40 \mathrm{~mA}$, were applied to examine the crystallinity of the samples.

Dissolution Study The in vitro dissolution test of the samples was done using the United States Pharmacopeia (USP) apparatus II (Verkin Dissolution Tester DIS 8000) in $900 \mathrm{ml}$ of distilled water. The paddle rotation was set at $200 \mathrm{rpm}$. The temperature was maintained at $37 \pm 0.5^{\circ} \mathrm{C}$. All the samples containing an equivalent amount of $360 \mathrm{mg}$ of ART were tested for their dissolution in water. The dissolved solution samples of $1 \mathrm{ml}$ were collected at $0.5,1,2,3$ and $4 \mathrm{~h}$ of dissolution time. For each sample, three dissolution tests were performed.

Analysis of ART Concentration Agilent 1100 series high performance liquid chromatography (HPLC) equipped with Kromasil C18 (150 mm $\times$ $4.6 \mathrm{~mm}$ i.d. $\times 3.5 \mu \mathrm{m})$ (Eka Chemicals AB, Sweden) column was used to determine the ART concentrations for the dissolution studies. ${ }^{15,16)}$ The mobile phase consisted of $75 \%$ of $0.01 \mathrm{~m}$ disodium hydrogen phosphate and $25 \%$ acetonitrile (HPLC grade) and the mobile phase was adjusted to $\mathrm{pH} 6.5$ with glacial acetic acid. The flow rate was set at $0.8 \mathrm{ml} / \mathrm{min}$. The detector was operated at a wavelength of $254 \mathrm{~nm}$.

The samples were filtered through $0.45 \mu \mathrm{m}$ polypropylene-reinforced Teflon membrane. The samples were subjected to pretreatment prior to injection into the HPLC system as ART is not very sensitive to UV. One milliliter of sample was added into $200 \mu 1$ of $10 \mathrm{M}$ sodium hydroxide and the mixture was heated at $45^{\circ} \mathrm{C}$ for $25 \mathrm{~min}$, which was then cooled to room temperature. Finally, $150 \mu$ of glacial acetic acid was added into the above mixture before injection into the HPLC system.

Phase Solubility Study The phase solubility study was performed using the method reported by Higuchi and Connors. ${ }^{17)}$ The concentrations of $\beta$-CD used were $0,2,4,6,8$ and $10 \mathrm{~mm}$. The vials were shaken continuously in a thermostatically controlled water bath at 24,37 and $52^{\circ} \mathrm{C}$ for $72 \mathrm{~h}$ until equilibrium was achieved. One milliliter sample of each solution was filtered through a $0.45 \mu \mathrm{m}$ polypropylene-reinforced Teflon membrane and diluted with water before HPLC analysis. The stability constants $K_{\mathrm{s}}$ at the three different temperatures were calculated from the linear section of the phase solubility diagrams using the following equation ${ }^{10)}$ :

$$
K_{\mathrm{s}}=\frac{\text { slope }}{S_{0}(1-\text { slope })}
$$

where $S_{0}$ is the intrinsic solubility of ART in the absence of $\beta$-CD, and the slope refers to the gradient of the plot of ART solubility (mM) vs. $\beta$-CD concentration (mM).

Furthermore, the enthalpy change $(\Delta H)$ for the $\beta$-CD-ART binary system was investigated using the the van't Hoff equation ${ }^{18}$ as shown below:

$$
\ln K_{\mathrm{s}}=-\frac{\Delta H}{R T}+\frac{\Delta S}{R}
$$

where $K_{\mathrm{s}}$ is as defined above, $T$ is the absolute temperature (Kelvin), $R$ is the gas constant $(8.314 \mathrm{~J} / \mathrm{mol} / \mathrm{K}), \Delta H$ is the enthalpy change, and $\Delta S$ is the entropy change. The enthalpy change $\Delta H$ was calculated from the slope of the plot of $\ln K_{\mathrm{s}} v s .1 / T$ after the least square linear regression analysis.

The Gibbs free energy of transfer $(\Delta G)$ of ART from pure water to aqueous solution of $\beta$-CD at each temperature was calculated using the following equation ${ }^{19)}$ :

$$
\Delta G=-R T \ln K_{\mathrm{s}}
$$

Statistical Analysis The percent dissolution efficiency (\%DE) for each sample was calculated as the percent ratio of area under the dissolution curve up to the time $t$, to that of the area of the rectangle described by $100 \%$ dissolution at the same time ${ }^{20)}$ as follows:

$$
\% \mathrm{DE}=\left(\frac{\int_{0}^{t} y \cdot d t}{y_{100} \cdot t}\right) \times 100
$$

The value of time for $75 \%$ of the drug to dissolve $\left(t_{75 \%}\right)$ was calculated using Sigma plot software. Mean dissolution time (MDT) was calculated by the following expression ${ }^{21)}$.

$$
\mathrm{MDT}=\frac{\sum_{i=1}^{n} t_{\mathrm{mid}} \Delta M}{\sum_{i=1}^{n} \Delta M}
$$

where $i$ is the dissolution sample number, $n$ is the number of dissolution times, $t_{\text {mid }}$ is time at the midpoint between times $t_{i}$ and $t_{i-1}$, and $\Delta M$ is the amount of ART dissolved $(\mu \mathrm{g})$ between times $t_{i}$ and $t_{i-1}$.

The similarity factor $\left.\left(f_{2}\right)^{22}\right)$ is given by:

$$
f_{2}=50 \times \log \left\{\left[1+\frac{1}{n} \sum_{i=1}^{n}\left|R_{i}-T_{i}\right|^{2}\right]^{-0.5} \times 100\right\}
$$

where $i$ is the dissolution sample number, $n$ is the number of dissolution times, $R_{i}$ and $T_{i}$ are the amount dissolved of the reference and the test drug sample at each time point $i$, respectively. It is 100 when the test and reference profiles are identical and approaches to 0 as the dissimilarity increases. 
The data were analyzed using a $t$-test for comparison between two different groups. The results were considered statically significant at $p<0.05$.

\section{Results}

Particle Morphology Scanning electron microscopy (SEM) photographs of the pure ART; ART nanoparticles and ART/ $\beta$-CD complexes prepared by EPN are shown in Fig. 3. It was observed from Fig. $3 b$ that the particle size of EPN prepared ART was in the range of $100-360 \mathrm{~nm}$ with the needle-like morphology. The particles of the pure ART (Fig. $3 \mathrm{a})$ were much larger $(5-50 \mu \mathrm{m})$ than those prepared by the EPN method. In addition, these particles lacked uniformity whereas the nanoparticles prepared by EPN were more uniform. In case of the ART/ $\beta$-CD complexes, the particle morphology is more blade or plate type as compared with the pure ART and its nanoparticles. It should be noted that the blade or plate type particles are of the free ART, which has not formed complex with $\beta-\mathrm{CD}$ as will be discussed in the FT-IR study.

FT-IR Study From Fig. 4a, the FT-IR spectrum of the pure ART showed the absorption peak at $1737 \mathrm{~cm}^{-1}$ due to the $\mathrm{C}=\mathrm{O}$ stretching vibrations of lactone and the absorption peaks in the range of $800-1200 \mathrm{~cm}^{-1}$ have been shown to be sensitive indicators of the $\mathrm{O}-\mathrm{O}$ and $\mathrm{C}-\mathrm{O}$ modes of the $\mathrm{O}-\mathrm{O}-\mathrm{C}$ (peroxide). The spectrum of $\beta-\mathrm{CD}$ (Fig. 4b) showed prominent peaks at $3450 \mathrm{~cm}^{-1}(\mathrm{O}-\mathrm{H}), 2923 \mathrm{~cm}^{-1}$ (C-H), $1642 \mathrm{~cm}^{-1}$ (H-O-H bending), $1155 \mathrm{~cm}^{-1}$ (C-O), and $1032 \mathrm{~cm}^{-1}$ (C-O-C). Shifts in the characteristic peaks, disappearance or decrease in intensity, and emergence of new peaks might be correlated to possible interactions between

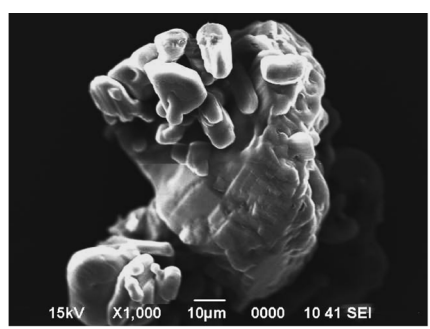

(a) Pure ART

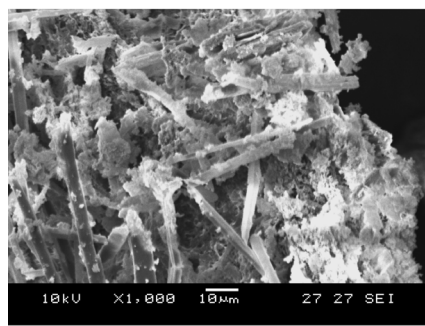

(c) ART/ $\beta-C D ~ 1: 1$

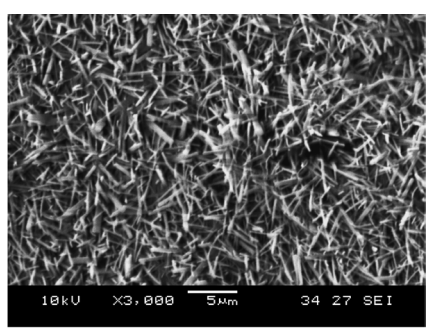

(b) ART nanoparticles

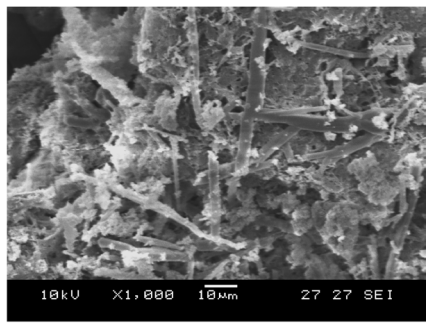

(d) ART/ $\beta$-CD 1:2

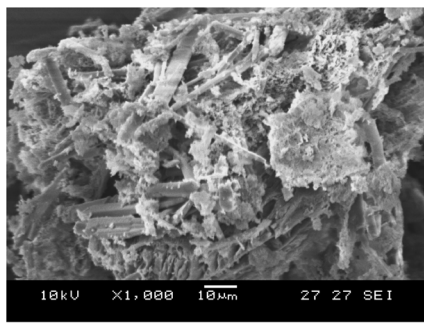

(e) ART/B-CD 1:4 the drug and $\beta$-CD. By comparing the spectra in Fig. $4 \mathrm{c}$, it can be noticed that the infrared spectrum of the physical mixture of ART and $\beta$-CD displays the unaltered characteristics of each component suggesting no or little interaction between ART and $\beta$-CD. On the contrary, in the case of ART/ $\beta$-CD complexes in Figs. $4 \mathrm{~d}-\mathrm{f}$, the peak at $1642 \mathrm{~cm}^{-1}$ shifted to a position between $1648 \mathrm{~cm}^{-1}$ and $1653 \mathrm{~cm}^{-1}$ as the concentration of $\beta$-CD increased in the complexes, indicating some interaction between the carbonyl group of the lactone in ART and $\beta$-CD. This result is in good agreement with the previous reports. ${ }^{23,24)}$ According to them, the shift of the carbonyl stretching is an evidence for the inclusion of the drug into the $\beta$-CD cavity. Therefore, the shift in the position of the absorption peaks implied the host-guest interaction and the formation of an inclusion complex from ART and $\beta$ $\mathrm{CD}$ in the particles prepared by EPN. This interaction is more pronounced for the $1: 4$ weight ratio ART/ $\beta$-CD complexes as seen in Fig. 4f. This interaction is not so evident at lower concentrations of $\beta-\mathrm{CD}$, as only some amount of ART forms complex with $\beta$-CD and the remaining is free ART which is not forming inclusion complex with $\beta-\mathrm{CD}$ as also supported by the SEM pictures in Fig. 3. The molar ratio corresponding to the weight ratio of $\mathrm{ART} / \beta-\mathrm{CD}$ is given in Table 1. As seen from Table 1, 1:4 weight ratio corresponds to the $1: 1$ molar ratio of $\mathrm{ART}: \beta$-CD, this shows the maximum complex formation at this weight ratio as compared to other lower weight ratios. At lower ART : $\beta$-CD weight ratios of $1: 1$ and $1: 2$, only some amount of ART forms complex with $\beta$-CD and the remaining is free ART.

X-Ray Diffraction The X-ray patterns of the orthorhombic, triclinic ART, the pure ART and, ART nanoparticles and ART/ $\beta$-CD complexes prepared by EPN are shown

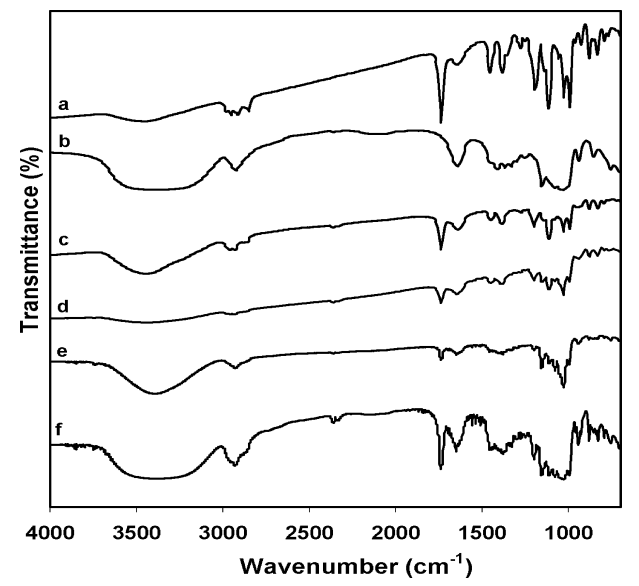

Fig. 4. FT-IR Spectra of (a) Pure ART, (b) $\beta$-CD, (c) ART/ $\beta$-CD Physical Mixture at $1: 1$ Ratio, (d) EPN Prepared ART/ $\beta$-CD Complex $(1: 1)$, (e) EPN Prepared ART/ $\beta$-CD Complex $(1: 2)$, and (f) EPN Prepared ART/ $\beta$ CD Complex $(1: 4)$

Table 1. Weight Ratios and Corresponding Molar Ratios for ART/ $\beta$-CD Complexes

\begin{tabular}{cc}
\hline \hline Weight ratio & Molar ratio \\
(ART $: \beta$-CD) & $($ ART $: \beta$-CD) \\
\hline $1: 1$ & $4.02: 1$ \\
$1: 2$ & $2.01: 1$ \\
$1: 4$ & $1.005: 1$ \\
\hline
\end{tabular}




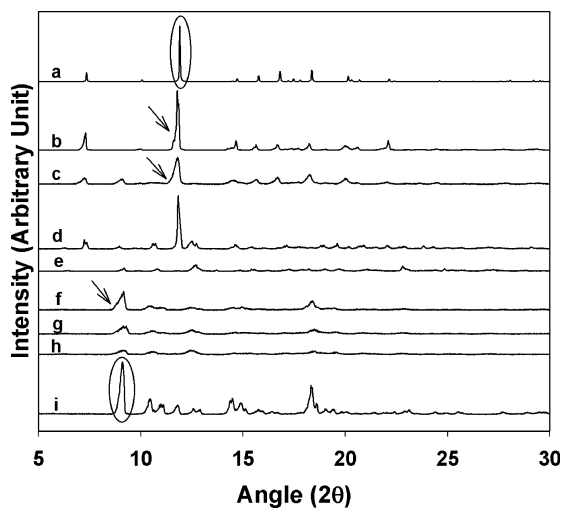

Fig. 5. X-Ray Diffractogram of (a) Orthorhombic Form or ART [from $\left.{ }^{13)}\right]$; (b) Pure ART; (c) EPN Prepared ART; (d) ART/ $\beta$-CD Physical Mixture (1:1), (e) $\beta$-CD, (f) EPN Prepared ART/ $\beta$-CD Complex $(1: 1)$, (g) ART/ $\beta$ CD Complex (1:2), (h) ART/ $\beta$-CD Complex (1:4); (i) Triclinic Form of ART [from ${ }^{13)}$ ]

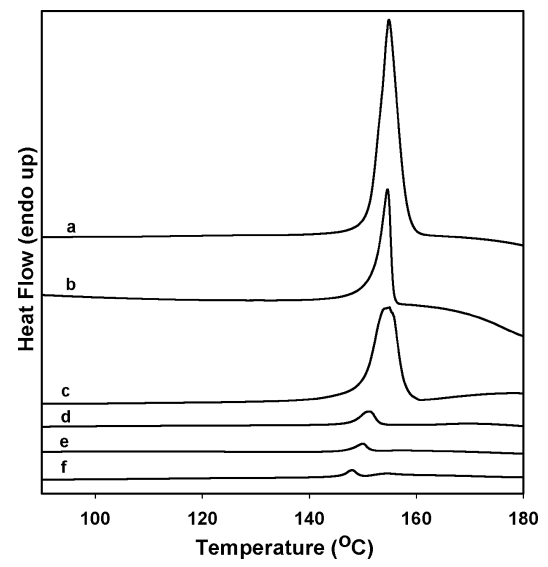

Fig. 6. DSC Thermogram of (a) Pure ART; (b) EPN Prepared ART; (c) ART $/ \beta$-CD Physical Mixture $(1: 1)$, (d) EPN Prepared ART/ $\beta$-CD Complex $(1: 1)$, (e) ART/ $\beta$-CD Complex $(1: 2)$, (f) ART/ $\beta$-CD Complex $(1: 4)$

Table 2. Melting Temperature $\left(T_{\mathrm{m}}\right)$, Heat of Fusion $\left(\Delta H_{\mathrm{f}}\right)$; Values of $t_{75 \%}$ and MDT (at $\left.4 \mathrm{~h}\right)$, Percent Dissolution Efficiency (\% DE) and Relative Dissolution (RD) for the Pure ART, EPN Prepared ART and ART/ $\beta$-CD Complexes, Corresponding Physical Mixture of ART/ $\beta$-CD at $1: 1$ Ratio

\begin{tabular}{lcccccrrr}
\hline \hline \multicolumn{1}{c}{ Sample } & $\begin{array}{c}\text { Melting temp. } \\
\left(T_{\mathrm{m}}\right)\end{array}$ & $\begin{array}{c}\Delta \mathrm{H}_{\mathrm{f}} \\
(\mathrm{J} / \mathrm{g})\end{array}$ & $\begin{array}{c}t_{75 \%} \\
(\mathrm{~h})\end{array}$ & $\begin{array}{c}\mathrm{MDT}_{4 \mathrm{~h}} \\
(\mathrm{~h})\end{array}$ & $\begin{array}{c}\% \mathrm{DE}_{0.5 \mathrm{~h}} \\
\% \mathrm{DE}_{4 \mathrm{~h}}\end{array}$ & $\mathrm{RD}_{0.5 \mathrm{~h}}$ & $f_{2}$ \\
\hline Pure ART & 156.0 & 76.20 & $>4$ & 1.163 & 2.5 & 9.29 & 1 \\
EPN ART & 154.0 & 59.18 & 3.86 & 1.108 & 10.43 & 49.89 & 4.17 \\
ART/ $\beta$-CD Phy Mix $(1: 1)$ & 155.7 & 71.83 & $>4$ & 1.118 & 3.68 & 13.40 & 1.47 \\
EPN ART/ $\beta$-CD 1: 1 & 148.0 & 22.05 & 0.82 & 0.790 & 27.94 & 81.93 & 11.17 \\
EPN ART/ $\beta$-CD 1:2 & 147.6 & 10.47 & 0.62 & 0.526 & 35.19 & 86.85 & 14.08 \\
EPN ART/ $\beta$-CD 1:4 & 145.2 & 7.91 & 0.43 & 0.328 & 43.84 & 91.79 & 17.53 \\
\hline
\end{tabular}

The similarity factor $\left(f_{2}\right)$ values at $(4 \mathrm{~h})$ for the test samples considering the pure ART as reference.

in Fig. 5. The orthorhombic form of ART in Fig. 5a displayed numerous distinct peaks at $2 \theta$ of $7.29^{\circ}, 11.78^{\circ}$, $14.65^{\circ}, 15.63^{\circ}, 16.64^{\circ}, 18.23^{\circ}, 20.0^{\circ}$, and $22.1^{\circ}$; whereas the triclinic form of ART in Fig. $5 \mathrm{i}$ exhibited peaks at $2 \theta$ of $9.24^{\circ}, 10.24^{\circ}, 10.79^{\circ}, 11.58^{\circ}, 14.56^{\circ}, 14.95^{\circ}$ and $18.01^{\circ}$. The pure ART was of the orthorhombic form as seen from Fig. 5b. The ART nanoparticles in Fig. 5c showed the similar diffraction pattern but with lower peak intensity, indicating the lower crystallinity of ART nanoparticles. The diffraction spectrum of $\beta$-CD in Fig. 5e does not show any prominent peak but extremely small peaks at $2 \theta=9.24^{\circ}, 12.5^{\circ}$ and $23.24^{\circ}$.

Comparing the EPN prepared ART/ $\beta$-CD complex at $1: 1$ ratio and the ART and $\beta$-CD physical mixture at the same ratio in Fig. $5 \mathrm{~d}$, it can be observed that the EPN prepared ART/ $\beta$-CD complex had the lower peak intensity than the ART and $\beta$-CD physical mixture at the same ratio, indicating that the decrease in the crystallinity was due to the formation of an amorphous form but not due to dilution by $\beta$-CD. This decrease in the XRD peak intensity is an evidence of the complexation of ART with $\beta$-CD. The ART/ $\beta$-CD complexes in Figs. $5 \mathrm{f}-\mathrm{h}$, exhibited the triclinic form of the drug with diffraction peaks at $2 \theta=9.24^{\circ}, 10.24^{\circ}$ and $10.79^{\circ}$; but with a lower peak intensity, suggesting a change from orthorhombic state to triclinic state and a decrease in the crystallinity of ART/ $\beta$-CD complexes during the EPN process. This indicates that besides forming an inclusion complex with ART (as suggested by FT-IR), the presence of $\beta$-CD during the EPN process also influenced the crystallization of the remaining ART from the solution into its triclinic form. The
XRD peaks in Figs. $5 \mathrm{f}-\mathrm{h}$ represent the free ART, which has not formed complex with $\beta$-CD. The peaks at $2 \theta$ of $9.24^{\circ}$, $10.24^{\circ}, 10.79^{\circ}$ and $18.01^{\circ}$ became lower in intensity as concentration of $\beta$-CD increased as more and more drug formed complex with $\beta-\mathrm{CD}$ and less amount of free drug was present in crystalline form. This indicates that the ART and $\beta$ $\mathrm{CD}$ formed complexes during the EPN process and the presence of $\beta$-CD during the EPN process affected the crystalline structure of the remaining ART that precipitated and did not form a complex with $\beta$-CD.

Thermal Properties DSC was conducted to understand the thermal properties of ART samples. As seen from Fig. $6 \mathrm{a}$, the pure ART showed a sharp melting endothermic peak at $156^{\circ} \mathrm{C}$. Being amorphous, $\beta$-CD showed a very broad melting endotherm in between 290 and $300^{\circ} \mathrm{C}$ (not shown). The endothermic melting peak of the EPN prepared ART in Fig. $6 \mathrm{~b}$ was slightly shifted to the lower temperature side. For all the ART/ $\beta$-CD complexes shown in Figs. $6 \mathrm{~d}-\mathrm{f}$, the ART endothermic peak shifted to a lower temperature compared to the pure ART and the EPN prepared ART, which indicated loss of crystallinity. The heat of fusion of the EPN prepared ART was lower than that of the pure ART. From Table 2, it is evident that the heat of fusion of the EPN prepared ART/ $\beta$ $\mathrm{CD}$ complexes decreased with increasing $\beta$-CD concentration. As heat of fusion is proportional to the crystallinity in the samples, these results suggested that the crystallinity of ART particles decreased when they were dispersed in the EPN prepared complexes. It can also be seen that the heat of fusion of the EPN prepared ART/ $\beta$-CD complex at $1: 1$ ratio was lower than those of the ART and $\beta$-CD physical mixture 


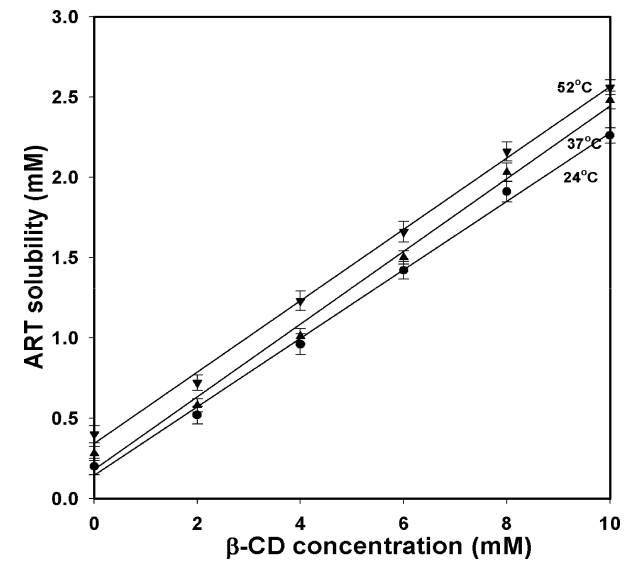

Fig. 7. Phase Solubility Diagram of $\beta$-CD-ART Binary System at $24^{\circ} \mathrm{C}$, $37^{\circ} \mathrm{C}$ and $52^{\circ} \mathrm{C}$

$\boldsymbol{\bullet}, 24^{\circ} \mathrm{C} ; \boldsymbol{\Lambda}, 37^{\circ} \mathrm{C}, \boldsymbol{\nabla}, 52^{\circ} \mathrm{C}$.

at the same ratio, indicating that the decrease in the crystallinity was due to the amorphous state formation and also due to complex formation between ART and $\beta$-CD. The shift in the melting peak of ART in the ART/ $\beta$-CD complexes also validates the formation of complex between ART and $\beta$-CD.

Phase Solubility Study The phase-solubility profile of ART in the presence of $\beta$-CD is shown in Fig. 7. The phase solubility of ART increased linearly with increasing $\beta$-CD concentration and temperature. The phase solubility curve could be classified as type $A_{\mathrm{L}}$ (linear), suggesting that the system was of first order in nature or formation of soluble $1: 1$ complexes. ${ }^{17)}$ The solubility increased approximately 11 fold for $\beta$-CD at the highest carrier concentration at $24^{\circ} \mathrm{C}$. The similar tendency was observed at $37^{\circ} \mathrm{C}$ and $52^{\circ} \mathrm{C}$. The related results have been observed for other drugs with $\beta$ $\mathrm{CD}$, attributed to the creation of weekly soluble complexes. The thermodynamic parameters $\left(\Delta G, \Delta H\right.$, and $\left.K_{\mathrm{s}}\right)$ are shown in Table 3. The correlation coefficient $\left(r^{2}\right)$ of van't Hoff plot was found to be 0.9915 , indicating a good fit. One can get information about the transfer process of ART from the pure water to an aqueous solution of the carrier from the values of Gibbs free energy change. Here, the Gibbs free energy change is a measure for the drug solubilisation in the aqueous carrier solution to be favourable or not. The negative $\Delta G$ values for all the carriers at different temperatures demonstrated the spontaneity of the ART solubilization process. The values of $\Delta G(\mathrm{~kJ} / \mathrm{mol})$ obtained at three different temperatures viz., 24,37 and $52^{\circ} \mathrm{C}$, were $-17.80,-17.92$, and -17.75 respectively. These values were not significantly different from the values $(-16.78,-16.81,-16.71 \mathrm{~kJ} / \mathrm{mol} \mathrm{re}-$ spectively) obtained in a similar phase solubility study done by Wong and Yuen. ${ }^{10)}$ The negative enthalpy $(\Delta H)$ indicated that the process was exothermic, i.e. energy was released which favoured the formation of the ART/ $\beta$-CD complex. The $\Delta H(\mathrm{~kJ} / \mathrm{mol})$ values of -18.41 and -17.54 were obtained in our phase solubility study and that by Wong and Yuen ${ }^{10)}$ indicating similarities in both the results.

Dissolution Studies Figure 8 shows the dissolution profiles of the pure ART, EPN prepared ART and ART/ $\beta$-CD complex. It is evident from Fig. 8a that the dissolution of the pure ART was very low (13\%) as compared to the EPN prepared ART (76\%) within $4 \mathrm{~h}$. The dissolution of ART from

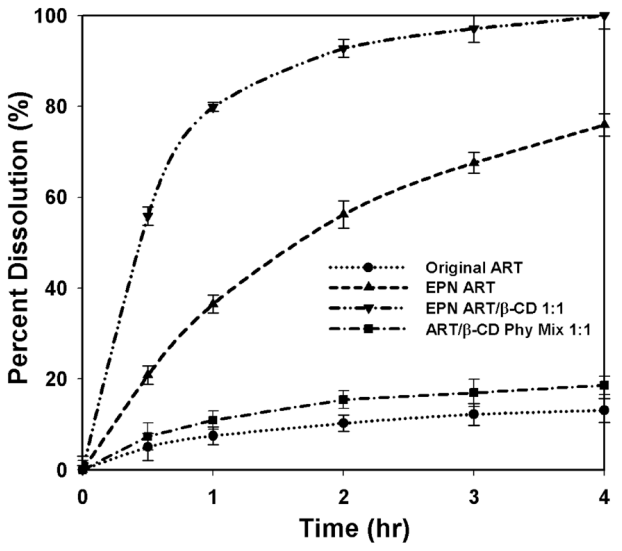

(a)

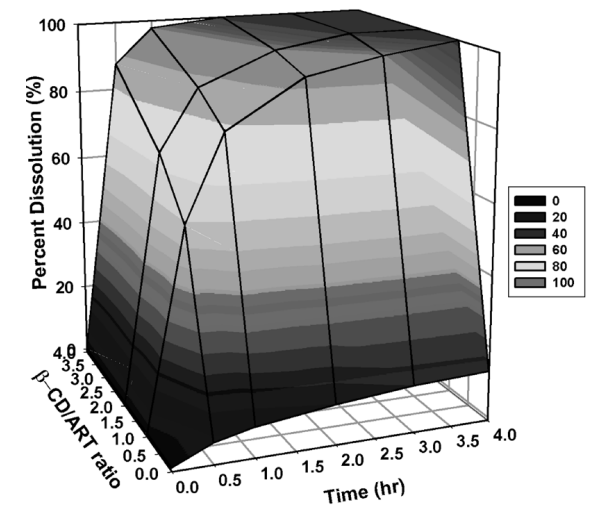

(b)

Fig. 8. (a) Dissolution Profiles of Pure ART; EPN Prepared ART, ART/ $\beta$ CD Complex and Physical Mixture at 1:1 Ratio, (b) 3D Dissolution Profile of ART/ $\beta$-CD Complexes with Respect to Pure ART

Table 3. Stability Constants $\left(K_{\mathrm{s}}\right)$ and Thermodynamic Parameter Values for the Solubilization Process of ART in Aqueous Solution of $\beta$-CD at $24^{\circ} \mathrm{C}$, $37^{\circ} \mathrm{C}$ and $52^{\circ} \mathrm{C}$

\begin{tabular}{cccc}
\hline \hline$T\left({ }^{\circ} \mathrm{C}\right)$ & $K \mathrm{~s}\left(\mathrm{M}^{-1}\right)$ & $\Delta \mathrm{G}(\mathrm{KJ} / \mathrm{mol})$ & $\Delta \mathrm{H}(\mathrm{KJ} / \mathrm{mol})$ \\
\hline 24 & 1355.66 & -17.80 & \\
37 & 1044.61 & -17.92 & -18.41 \\
52 & 713.78 & -17.75 & \\
\hline
\end{tabular}

the physical mixture of ART and $\beta$-CD at $1: 1$ ratio was almost the same as that from the pure ART and was much lower than the ART from the EPN prepared ART/ $\beta$-CD complex at $1: 1$ ratio $(100 \%)$ in $4 \mathrm{~h}$. The $3 \mathrm{D}$ dissolution profile of the EPN prepared ART/ $\beta$-CD complexes with respect to the pure ART, with varying $\beta$-CD to ART ratio, can be seen from Fig. 8b, which demonstrated that the drug dissolution increased with increasing the concentration of the water-soluble carrier $\beta$-CD.

The dissolution data obtained were statistically analyzed for more comprehensive understanding. It can be seen from Table 2 that the pure ART and ART/ $\beta$-CD physical mixture at $1: 1$ ratio did not even reach $75 \%$ dissolution within $4 \mathrm{~h}$. The EPN prepared ART reached $75 \%$ dissolution in $3.86 \mathrm{~h}$. For the EPN prepared ART complexes, the time for $75 \%$ dissolution was shorter than that for the EPN prepared ART. Also, $t_{75 \%}$ of the EPN prepared ART complexes decreased 
with increasing $\beta$-CD concentration in the complexes. It was verified that the dissolution of the ART was significantly influenced by the water-soluble carrier, and the calculated mean dissolution time (MDT) values for all the samples investigated also supported this finding. The MDT indicates the time for the drug to dissolve for the cumulative dissolution process. ${ }^{25)}$ The lower the MDT value, the faster the dissolution is. For the pure ART, MDT value was very high $(1.16 \mathrm{~h})$. This value decreased after preparing ART by the EPN process and decreased drastically for the ART/ $\beta$-CD complexes, decreasing with increasing carrier concentration in the complexes.

It was also observed from Table 2 that the value of dissolution efficiency at $0.5 \mathrm{~h}\left(\% \mathrm{DE}_{0.5 \mathrm{~h}}\right)$ was enhanced from 2.5 for the pure ART to 10.43 for the EPN prepared ART to a high 43.84 for the EPN prepared ART/ $\beta$-CD complex at $1: 4$ ratio. Similar to $\% \mathrm{DE}_{0.5 \mathrm{~h}}$ values, the value of the relative dissolution at $0.5 \mathrm{~h}\left(\mathrm{RD}_{0.5 \mathrm{~h}}\right)$ was high for the EPN prepared ART/ $\beta$-CD complexes. $\% \mathrm{DE}_{0.5 \mathrm{~h}}$ and $\mathrm{RD}_{0.5 \mathrm{~h}}$ values (3.68 and 1.47 , respectively) for the physical mixture of ART and $\beta$ $\mathrm{CD}$ at the same ratio of $1: 1$ were much lower than those of the EPN prepared ART/ $\beta$-CD complexes at $1: 1$ ratio (27.94 and 11.17 , respectively). The highest $\% \mathrm{DE}_{4 \mathrm{~h}}$ was 91.79 for the EPN prepared ART/ $\beta$-CD complexes at the highest $\beta$-CD concentration, which also revealed the highest improvement in dissolution rate of ART. Furthermore, comparison between the dissolution profiles of ART from different formulations was made using the similarity factor $\left(f_{2}\right)$ considering the pure ART as reference. According to the FDA's guidelines $f_{2}$ values greater than 50 show the similarity of the dissolution profiles. ${ }^{22)}$ From Table 2, it was observed that the dissolution profile of the fabricated samples (i.e. EPN prepared ART, $\mathrm{ART} / \beta$-CD complexes) and the pure ART were not at all similar as the $f_{2}$ values for all these cases were lower than 50 . But in the case of the physical mixture of ART/ $\beta$-CD at $1: 1$ ratio, $f_{2}$ value was greater than 50 , showing a high similarity. Comparison between two different groups using a $t$-test also showed the statically significant differences between pure ART and all the EPN prepared samples (ART nanoparticles, $\mathrm{ART} / \beta$-CD complexes) at $p<0.05$.

\section{Discussion}

The Noyes-Whitney equation provides an indication about how the dissolution rate of an insoluble drug might improve. ${ }^{26)}$ According to it, the dissolution velocity is proportional to the concentration gradient and is a function of the particle surface area amongst other things:

$$
\frac{d c}{d t}=\frac{D \cdot A}{h}\left(C_{\mathrm{S}}-C\right)
$$

where $d c / d t$ is the rate of dissolution, $D$ is the diffusion coefficient, $A$ is the surface area of the solid, $h$ is the diffusion layer thickness, $C_{\mathrm{S}}$ is the saturation solubility of the drug and $C$ is the concentration of the solid in the bulk dissolution medium. According to this equation, reducing the particle size to nanoscale will result in an increased particle surface area, which in turn will lead to an enhanced dissolution rate of the drug. The particle size of pure ART was reduced to a size in a range of approximately $100-360 \mathrm{~nm}$ by the EPN method, which led to the increased dissolution of ART as compared to the pure ART. In addition to that, the degree of crystallinity also controls the dissolution rate of a drug. A drug in the amorphous or metastable state possesses a faster dissolution rate due to its higher internal energy and greater molecular motion. $\left.{ }^{9}, 10\right)$ The lower crystallinity of ART nanoparticles, as indicated by the XRD and DSC studies, also contributed to the improvement in the dissolution rate of ART.

The polymorphic form of a drug can have a considerable effect on its pharmaceutical effectiveness and its physical properties like the dissolution rate etc. As mentioned earlier, Chan et al. have reported the X-ray crystallographic verification for the two ART polymorphs-triclinic and orthorhombic polymorphs. ${ }^{13)}$ ART crystals of one polymorph have unit cell with dimensions of $a=9.450 \AA, b=24.090 \AA, c=6.364 \AA$ corresponding to an orthorhombic system of $P 2_{1} 2_{1} 2_{1}$ space group. ${ }^{13)}$ The other polymorph has cell dimensions of $a=9.891 \AA, b=15.343 \AA, c=9.881 \AA ; \alpha=90.92^{\circ}, \beta=102.99^{\circ}$, $\gamma=93.28^{\circ}$ corresponding to a triclinic system of a space group $P 1{ }^{13)}$ There are other physicochemical differences between these two polymorphs based on their different crystal structures. The orthorhombic form of ART possesses greater density and lower solubility in water than the triclinic form. The orthorhombic crystal has a denser and thicker appearance like rods and prisms, whereas the triclinic form mainly contained thin blades and plates. The orthorhombic form of ART has been reported more often ${ }^{13,27)}$ than the triclinic form. ${ }^{13)}$

In this study we have fabricated both the polymorphs of ART. As can be seen from the X-ray diffractogram in Fig. 5, the pure ART and the EPN prepared ART nanoparticles exhibited the orthorhombic form of the drug. The physical mixture of the pure ART and the $\beta$-CD carrier displayed the peaks of the orthorhombic form of ART and the $\beta$-CD carrier. However, the X-ray patterns of the free ART in all the ART/ $\beta$-CD complexes prepared under the same conditions as the ART nanoparticles presented the triclinic form of ART. The different morphology of the two polymorphs can also be seen from the SEM photographs in Fig. 3. The pure ART had the prism and rod type morphology and the EPN prepared particle showed the needle-like morphology, whereas free ART in all the ART/ $\beta$-CD complexes had the blade and plate type morphology. This means that during the EPN process, the ART crystallized into the triclinic form in the presence of $\beta$-CD. The addition of $\beta$-CD affected the crystallization process and induced some changes in the crystal structure of ART which resulted in the formation of a triclinic form. As previously reported, the conformation of both orthorhombic and triclinic molecules of artemisinin and their bond lengths are quite similar, whereas the bond angles and the torsion angles are different for the two polymorphs. ${ }^{13)}$ It is predicted that the presence of $\beta$-CD must have altered the bond angle and torsion angle, resulting in the formation of a triclinic form of ART in the ART/ $\beta$-CD complexes prepared by EPN.

In the FT-IR spectra, the shift in the absorption peaks was related to possible interaction between ART and $\beta$-CD and the formation of ART/ $\beta$-CD complexes. The phase solubility studies also confirmed the formation of the soluble $1: 1 \mathrm{com}$ plexes. The molar ratio corresponding to the weight ratio of ART/ $\beta$-CD is given in Table 1 . As seen from Table $1,1: 4$ weight ratio corresponds to the $1: 1$ molar ratio of ART: $\beta$ $\mathrm{CD}$, this shows the maximum complex formation at this 
weight ratio as compared to other lower weight ratios. The lower peak intensity in the XRD study and the lower melting point with the reduced heat of fusion indicated by the DSC study also implies the formation of ART/ $\beta$-CD complexes and the presence of the amorphous form of the drug. The ability of $\beta$-CD to form a complex with ART has also been reported earlier ${ }^{10,28)}$ and the properties of ART as a drug appear to be unaffected upon complexation with $\beta$-CD. ${ }^{28)}$

From the analysis of the dissolution data, we can predict that the dissolution of ART from the EPN prepared ART/ $\beta$ $\mathrm{CD}$ complexes was higher due to formation of the inclusion complex with $\beta$-CD, formation of the triclinic form for the remaining free ART (not complexed with $\beta$-CD), and amorphous state formation. Besides the formation of a complex, as the triclinic form of ART has a higher solubility, therefore, all the ART/ $\beta$-CD complexes presented the improved dissolution properties over the pure orthorhombic ART and also the EPN prepared ART nanoparticles. Therefore, it can be concluded that our EPN prepared ART nanoparticles and ART/ $\beta$-CD complexes have much faster dissolution than the pure ART and this enhanced dissolution can be translated into an improved bioavailability of the drug after oral administration.

\section{Conclusion}

This study revealed that the EPN method can successfully fabricate ART nanoparticles and ART/ $\beta$-CD complexes to significantly improve dissolution of ART from its pure form. The dissolution rate of ART particles is affected by the particle size, degree of crystallinity and polymorphic form of the drug. The pure ART and EPN prepared ART nanoparticles presented the orthorhombic form of the drug whereas the free ART in ART/ $\beta$-CD complexes had the triclinic form. The improved dissolution of ART/ $\beta$-CD complexes was attributed to the formation of the inclusion complex with $\beta$ $\mathrm{CD}$, formation of the triclinic form for the remaining free ART (not complexed with $\beta$-CD), and amorphous state formation. The ART nanoparticles and ART/ $\beta$-CD complexes prepared by the fabrication method described in this study would have a high potential for delivery in much smaller doses compared with commercial preparation containing the normal form of the drug.

Acknowledgement The authors acknowledge the financial support from the Lee Kuan Yew Postdoctoral Fellowship, SUG M58050023, Nanyang Technological University, Singapore. The authors also acknowledge Dr. Po- ernomo Gunawan from School of Chemical and Biomedical Engineering (Nanyang Technological University) for his help with the X-ray diffraction.

\section{References}

1) Balint G. A., Pharmacol. Ther, 90, 261-265 (2001).

2) Dhingra V., Rao K. V., Narasu M. L., Life Sci., 66, 279-300 (2000).

3) Oh S., Kim B. J., Singh N. P., Lai H., Sasaki T., Cancer Lett., 274, 33-39 (2009).

4) Marconi G., Monti S., Manoli F., Esposti A. D., Guerrini A., Helv. Chim. Acta, 87, 2368-2377 (2004).

5) Subramaniam B., Rajewski R. A., Snavely K., J. Pharm. Sci., 86, 885-890 (1997).

6) Hancock B. C., Zografi G., J. Pharm. Sci., 86, 1-12 (1997).

7) Grau M. J., Kayser O., Muller R. H., Int. J. Pharm., 196, 155-157 (2000).

8) Wong J. W., Yuen K. H., Int. J. Pharm., 227, 177-185 (2001).

9) Sharma U. S., Balasubramanian S. V., Straubinger R. M., J. Pharm. Sci., 84, 1223-1230 (1995).

10) Wong J. W., Yuen K. H., Drug Dev. Ind. Pharm., 29, 1035-1044 (2003).

11) Kakran M., Sahoo N. G., Li L., Judeh Z., J. Pharm. Pharmacol., 62, 413-421 (2010).

12) Kakran M., Sahoo N. G., Li L., Judeh Z., Panda P., J. Biomater. Sci. Polym. Ed., 22, 363-378 (2011).

13) Chan K. L., Yuen K. H., Takayanagi H., Janadasa S., Peh K. K., Phytochemistry, 46, 1209-1214 (1997).

14) Kakran M., Sahoo N. G., Li L., Judeh Z., Wang Y., Chong K., Loh L., Int. J. Pharm., 383, 285-292 (2010).

15) Zhao S. S., Analyst, 112, 661-664 (1987).

16) Sahoo N. G., Abbas A., Judeh Z., Li C. M., Yuen K. H., J. Pharm. Sci., 98, 281-296 (2009).

17) Higuchi T., Connors K. A., Adv. Anal. Chem. Instr., 4, 117-212 (1965).

18) Martin A., "Physical Pharmacy, Physical Chemical Principles in the Pharmaceutical Sciences," 4th ed., Lea and Febiger, Philadelphia, 1993.

19) Ahuja N., Katare O. P., Singh B., Eur. J. Pharm. Biopharm., 65, 26 38 (2007).

20) Khan K. A., J. Pharm. Pharmacol., 27, 48-49 (1975).

21) Costa F. O., Sousa J. J. S., Pais A. A. C. C., Formosinho S. J., J. Controlled Release, 89, 199-212 (2003).

22) Costa P., Lobo J. M. S., Eur. J. Pharm. Sci., 13, 123-133 (2001).

23) Hussein K., Türk M., Wahl M. A., Pharm. Res., 24, 585-592 (2007).

24) Jensen C. E., dos Santos R. A., Denadai A. M., Santos C. F., Braga A N., Sinisterra R. D., Molecules, 15, 4067-4084 (2010).

25) Reppas C., Nicolaides E., "Oral Drug Absorption Prediction and Assessment," ed. by Dressman J. B., Lennernas H., Marcel Dekker, New York, 2000, pp. 229-254.

26) Noyes A. A., Whitney W. R., J. Am. Chem. Soc., 19, 930-934 (1897).

27) Lisgarten J. N., Potter B. S., Bantuzeko C., Palmer R. A., J. Chem Crystallogr., 28, 539-543 (1998).

28) Marconi G., Monti S., Manoli F., Esposti A. D., Mayer B., Chem Phys. Lett., 383, 566-571 (2004). 